Fortner, J.G., Booher, R.J. \& PACK, G.T. (1964) Results of groin dissection for malignant melanoma. Surgery, 55, 485.

Gough, M.H., GuINeY, E.G. \& KinmonTH, J.B. (1963) Lymphangiography: new techniques and uses. Brit. med. $J . \mathbf{i}, 1181$.

JANTET, G.H. (1958) 9th Scientific Meeting, Surgical Research Society, London.

JANTET, G.H., KINMONTH, J.B., EdwaRds, J.M. \& Gough, M.H. (1964) Endolymphatic therapy with radioactive gold for malignant melanoma. Brit. med.J. ii, 904.

Kinmonth, J.B. (1965) Lymphoedema of lower limb. Proc. roy. Soc. Med. 58, 1021.

Kinmonth, J.B. (1952) Lymphangiography in man. Clin. Sci. 11, 13.
Kinmonth, J.B., Rob, C.G. \& Simeone, F.A. (1962) Vascular Surgery. Arnold, London.

Lemon, W.T., Ketcham, A.S., McLowry, J.D. \& Herdt, J. (1966) Surgical applications of ethiodol with chlorophyll in lymphangiography. Ann. Surg. 164, 114.

RAVEL, R. (1966) Histopathology of lymph nodes after lymphangiography. Amer. J. Clin. Path. 46, 335.

Shibata, H.R., McLean, P., Vezina, J.C., Inglis, F.G. \& TABAH, E.J. (1966) Axillary lymphography in carcinoma of breast. Surgery, 60, 329.

Wallace, S., Jackson, L., Schaffer, B., Gould, J., Greening, R.R., Weiss, A. \& Kramer, S. (1961) Lymphangiograms: their diagnostic and therapeutic potential. Radiology, 76, 179.

\title{
Muscle necrosis and acute renal failure in carbon monoxide
} poisoning

\author{
A. L. Linton \\ M.B., M.R.C.P.(Ed.), \\ M.R.C.P.(Glas.) \\ Consultant Physician \\ D. H. LAWSON \\ J. Hume Adams \\ M.B., Ph.D., M.C.Path. \\ M.B., M.R.C.P.(Ed.) \\ Senior Lecturer in Neuropathology \\ Medical Registrar
}

Renal Unit and Department of Pathology, Western Infirmary, Glasgow

SCATTERED reports have appeared in the literature concerning the occurrence of acute muscle necrosis in cases of carbon monoxide and barbiturate poisoning (Howse \& Seddon, 1966). Acute renal failure, presumably due to the release of myoglobin from the necrosed muscle cells, has been reported in such a case by Loughridge, Leader \& Bowen (1958). The present case is reported because of the relative rarity of the condition, because of the diagnostic problems involved and because the patient survived the additional complication of acute renal failure.

\section{Case report}

A 44-year-old female was admitted on 11 October 1966 having taken an unknown number of capsules of sodium amytal and imipramine, and having then attempted to gas herself. On admission she was comatose and arreflexic; shortly afterwards she developed apnoea and severe hypotension. Carboxyhaemoglobin level was $35 \%$, and serum barbiturate level $1.8 \mathrm{mg} / 100 \mathrm{ml}$. She was treated with the usual conservative measures, artificial ventilation and forced diuresis, as described by Linton, Luke \& Briggs (1967). She regained consciousness $12 \mathrm{hr}$ after admission, and there were no cerebral or respiratory complications. It was then noted that her urine volume had fallen to $5 \mathrm{ml} / \mathrm{hr}$, although this had been excellent during the $10 \mathrm{hr}$ of forced diuresis. The urine was dark brown in colour, and was initially reported to contain haemoglobin. It was suspected that the patient had sustained an episode of acute intravascular haemolysis probably as a result of drug ingestion. The diagnosis of acute renal failure was made on the basis of continuing severe oliguria (urine volume $<300 \mathrm{ml} /$ day), a blood urea of $180 \mathrm{mg} /$ $100 \mathrm{ml}$ and a urinary urea concentration of $800 \mathrm{mg} / 100 \mathrm{ml}$. The usual conservative regime for acute renal failure was established, but despite severe fluid restriction the patient developed gross oedema of both legs and feet about 5 days after admission. This feature, together with the marked tenderness of the leg muscles, and the fact that the brown pigment disappeared very quickly from the serum despite severe renal impairment raised the possibility that this was a case of muscle necrosis and that the pigment was myoglobin, which has a much higher renal clearance than haemoglobin (Bailie, 1964). That the urinary pigment was in fact myoglobin was then confirmed by spectroscopic examination.

The patient remained severely oliguric for 15 days, and required one peritoneal dialysis and two haemodialyses before diuresis occurred. She then made an uncomplicated recovery from her renal failure, but when mobilization was attempted, she had great difficulty in walking. Examination of her legs revealed that in addition to oedema, her calf muscles were tense, 
her feet were fixed in plantar flexion, and there were bilateral contractures of the tendo Achillis. She complained of paraesthesiae of the feet, and patchy sensory loss was found over the soles and lateral borders of her feet. Electro-myography showed signs of severe partial denervation, and serial tests demonstrated progressive improvement. Serum creatinephosphokinase level was 14.5 i.u./1, which was within normal limits and did not suggest an acute myopathy. After orthopaedic consultation ( $\mathrm{Mr}$ T. B. Gardiner) it was agreed that the patient was suffering from bilateral ischaemic contractures involving principally soleus and gastrocnemius, with a resulting incapacitating equinus deformity. Operation for bilateral lengthening of the Achilles tendons was therefore undertaken and muscle biopsies were obtained. The results of this corrective procedure have been excellent, and the patient can now walk normally. She has regained good power in her calves and can stand on tiptoe on either foot. Her renal function is also normal with a creatinine clearance of $120 \mathrm{ml} / \mathrm{min}$.

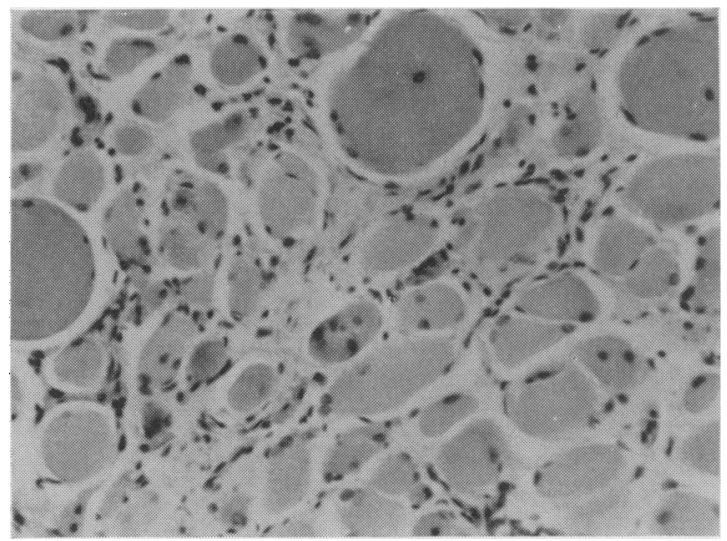

FIG. 1. Soleus. Note great variation in fibre diameter, central nuclei and moderate proliferation of sarcolemmal nuclei. There is only a mild increase of cells in the interstitial tissue. Haematoxylin and eosin, $\times 100$.

\section{Histology}

Biopsy specimens were obtained from soleus, flexor digitorum longus, flexor hallucis longus and tibialis posterior on the right, and from the left soleus. These were sectioned and stained with haematoxylin and eosin, haematoxylin and van Gieson's fluid, phosphotungstic acid haematoxylin and by Masson's trichrome method.

1. Soleus. The most striking abnormality was the great variation in fibre diameter, many of the larger fibres containing central nuclei (Fig. 1);

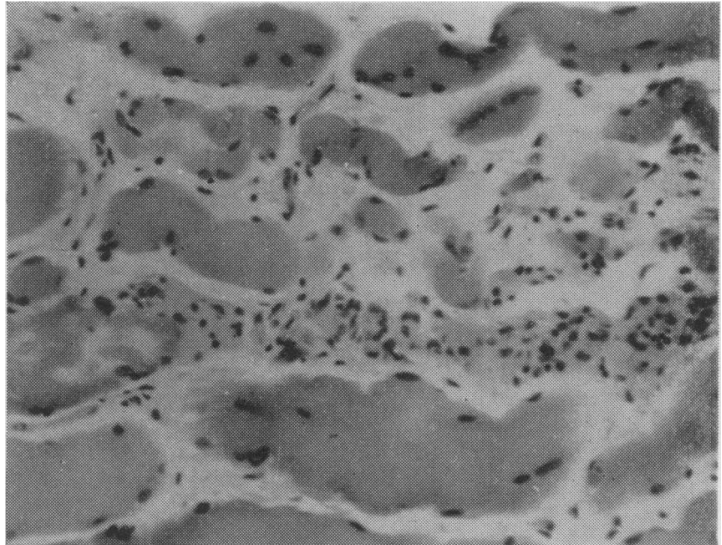

Fig. 2. Soleus. One muscle fibre is undergoing active phagocytosis. There is also coarse vacuolation in a fibre and mild 'chaining' of sarcolemmal nuclei. H. \& E., $\times 100$.

a few showed obvious degenerative changes with coarse vacuolation or intense eosinophilia with loss of cross striations. Occasional fragmented fibres were undergoing active phagocytosis (Fig. 2). The great majority of sarcolemmal nuclei were of normal appearance. However, occasional nuclei related to small fibres, some of which were intensely basophilic (Fig. 3), and nuclei in the numerous abortive multi-nucleated buds throughout the muscle were large and contained prominent nucleoli. There were only short chains of sarcolemmal nuclei. In the interstitial tissue there was no conspicuous increase of collagen, fat or lymphocytes.

2. Flexor hallucis longus. In contrast to soleus, there was a great increase in interstitial collagen

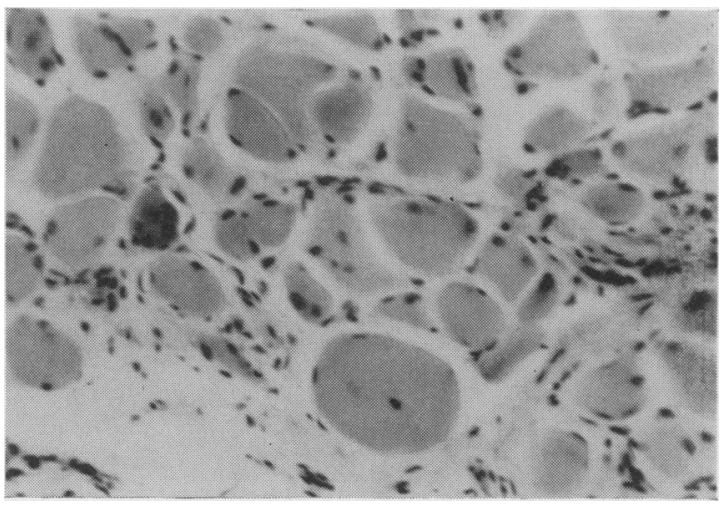

FIG. 3. Soleus. Note the small multinucleate basophilic fibre. H. \& E., $\times 100$. 


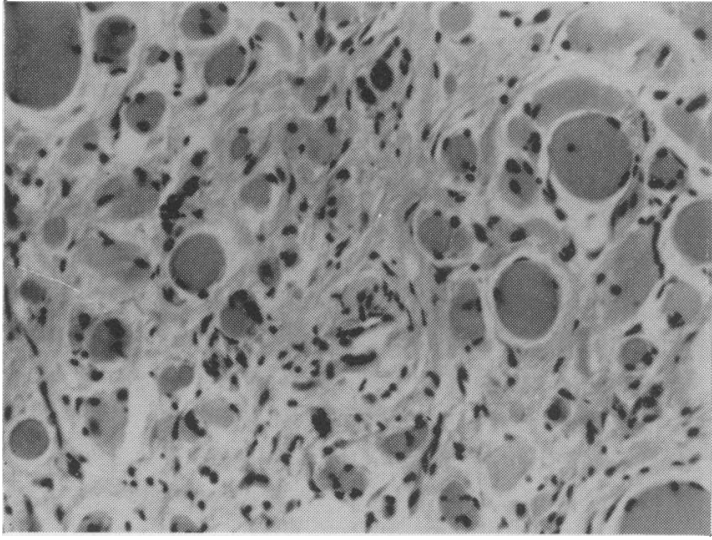

FIg. 4. Flexor hallucis longus. In addition to great variation in fibre diameter, there is a marked increase of interstitial collagen. H. \& E., $\times 100$.

(Fig. 4). There were numerous residual muscle fibres throughout the fibrous tissue, showing degenerative changes similar to those in soleus. There was no evidence of a frank infarct. The fibrous tissue was locally infiltrated with lymphocytes and occasional plasma cells (Fig. 5).

3. Flexor digitorum longus. Muscle fibres showed the same abnormalities as in flexor hallucis longus, and there was a moderate increase of interstitial collagen.

4. Tibialis posterior. This muscle was least severely affected, but a few fibres showed changes similar to those observed in soleus.

Vessels and small nerves within all the muslces seemed normal. No large nerve trunk was identified.

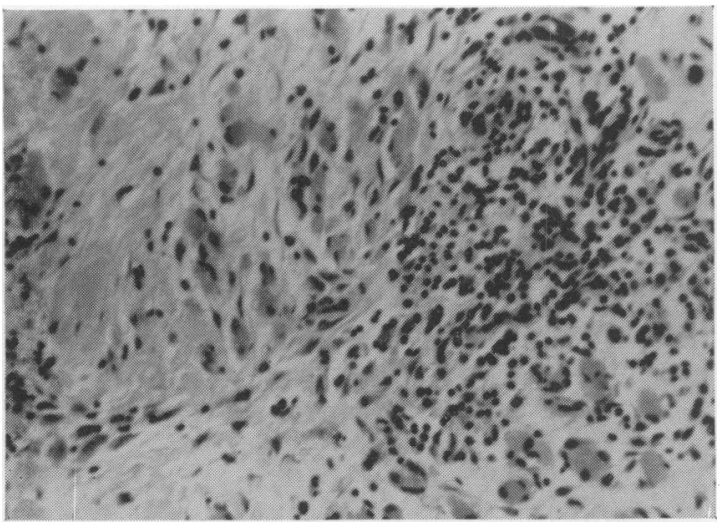

FIg. 5. Flexor hallucis longus. Interstitial fibrosis is dense and there is a conspicuous infiltrate of lymphocytes. H. \& E., $\times 100$.

\section{Discussion}

Howse \& Seddon (1966) reported on the occurence of muscle necrosis after carbon monoxide and barbiturate poisoning, and the condition is thought to result from a combination of general hypoxia due to impaired respiration or the presence of carboxyhaemoglobin together with effects of pressure reducing blood-flow to a limb and localizing the lesion. Their patients all had a condition resembling Volkmann's ischaemic contracture in an upper limb, attributed to pressure of the patient's head on the arm during unconsciousness. Our patient who had a bilateral lesion of the lower legs, was found with her legs over a raised fender, which was pressing on the upper posterior aspect of the calves, and this supports the view that pressure localizes the ischaemic lesion.

The clinical problems presented by this patient concerned the diagnosis and treatment of the ischaemic muscle lesion. After the apnoea and hypotension had been dealt with, her recovery of consciousness was rapid. The appearance of dark brown urine and increasingly severe oliguria in a patient who has ingested an overdose of drugs will naturally lead to a suspicion that intravascular haemolysis has occurred. As simple chemical tests of the urinary pigment may not differentiate between haemoglobin and myo globin, absorption spectrophotometry should be carried out. Rapid disappearance of the pigment from the serum despite oliguria suggests the correct diagnosis; haemoglobin, with a much lower renal clearance than myoglobin, will persist much longer in the serum. Clinically, the appearance of swollen, tender muscles may suggest the diagnosis, but in our case these were not obvious for 2-3 days after the acute incident.

Acute renal failure of moderately severe degree followed rapidly on the episode of myoglobinuria, due presumably to the known toxic effect of myoglobin on the tubules (Tavill et al., 1964). It is conceivable that the episode of hypotension after drug ingestion was also a contributory factor, but a good urine flow was maintained by forced diuresis for $12 \mathrm{hr}$ after correction of hypotension. It seems most likely that myoglobinuria was, the main cause of the acute tubular necrosis. Loughridge et al. (1958) recorded a similar, but fatal, case, and the present patient is the first such case recorded with survival; her renal function is apparently now completely normal.

The clinical appearances of the ischaemic lesion are of mixed muscle and nerve damage (Howse \& Seddon, 1966). In this case, electromyographic studies were more suggestive of 
partial denervation than of muscle necrosis, but pathological examination of muscle revealed no evidence of neurogenic atrophy. The electromyographic changes and the clinical evidence of nerve damage improved fairly quickly, and the residual problem was an orthopaedic one, corrected by bilateral lengthening of tendo Achillis, with excellent functional result.

Histological studies of the affected muscles were of considerable interest. In the severely affected right soleus, the variation in fibre diameter with large swollen fibres, eosinophilic fibres and small basophilic fibres, together with necrotic fibres, coarse vacuolar degeneration, central nuclei, phagocytosis and many muscle giant cells were suggestive of an active, progressive degenerative process such as a muscular dystrophy. However, serum enzyme studies, biopsy of an unaffected triceps muscle and the subsequent clinical progress rule out this possibility. In marked contrast to soleus, the flexor hallucis longus showed advanced interstitial fibrosis and a dense infiltration of leucocytes. The other muscles show changes intermediate between those two extremes. In this case there was no evidence of a frank muscle infarct, although Howse \& Seddon (1966) describe changes indistinguishable from Volkmann's ischaemic contracture. Bowden \& Gutmann (1949) showed that several conditions may produce contracture in voluntary muscles; the appearances of flexor hallucis longus in the present case are very similar to the group described as 'diffuse interstitial fibrosis' by these authors. The same type of fibrosis may follow venous occlusion (Adams, Denny-Brown
\& Pearson, 1962, Chap. 9), and the same authors (Chapter 3) state that affected muscles in carbon monoxide poisoning may show a variety of degenerative changes. The changes are very similar to those seen in the 'anterior tibial syndrome' which is also thought to be due to ischaemia. It may be concluded that the abnormalities observed in the present case simply demonstrate the wide variety of changes which may occur in muscle partly or wholly deprived of oxygen.

\section{Acknowledgments}

We wish to thank Dr W. S. Thompson for assaying this patient's serum creatinephosphokinase, Dr I. D. Melville for performing the electromyogram and $\mathrm{Mr} \mathrm{T}$. B. Gardiner for undertaking the necessary orthopaedic procedures.

\section{References}

Adams, R.D., Denny-Brown, D. \& Pearson, C.M. (1962) Diseases of Muscle, 2nd edn. Henry Kimpton, London.

BaILIE, M.D. (1964) Primary paroxysmal myoglobinuria. New Engl. J. Med. 271, 186.

Bowden, R.E.N. \& Gutmann, E. (1949) The fate of voluntary muscle after vascular injury in man. J. Bone Jt Surg. 31B, 356.

Howse, A.J.G. \& Seddon, Sir Herbert (1966) Ischaemic contracture of muscle associated with carbon monoxide and barbiturate poisoning. Brit. med. J. i, 192.

Linton, A.L., LuKe, R.G. \& BRIGGS, J.D. (1967) Methods of forced diuresis and its application in barbiturate poisoning. Lancet, ii, 377.

LOUghridge, L., Leader, L. \& BOWEN, D.A.L. (1958). Acute renal failure due to muscle necrosis in carbon monoxide poisoning. Lancet, ii, 349.

Tavill, A.S., Evanson, J.M., Baker, S.B. \& HewitT, V. (1964) Idiopathic paroxysmal myoglobinuria with acute renal failure and hypercalcaemia. New Engl. J. Med. 271, 283.

\title{
Bilateral retrobulbar neuritis due to insecticides*
}

\author{
H. R. JINDAL $\dagger$ \\ F.R.C.S. \\ Chester Royal Infirmary, Chester
}

INSECTICIDES are very important in national economies, especially those of the developing countries. Their uses are too well-known to enumerate here. The object of this paper is to record a toxic effect which has received insufficient attention. This is the case of a patient who had been spraying a certain proprietary pre-

\footnotetext{
*This case was presented at the North of England Ophthalmological Society Meeting for discussion. head.

†Present address: Birkenhead General Hospital, Birken-

paration to kill woodworm and developed retrobubor neuritis. The preparation contained dieldrin and pentachlorophenol in petroleum distillate.

In 1952, Campbell described three cases of monocular retrobulbar neuritis that occurred in a man and two women who had been using an insecticide preparation for treating woodworm. In each patient the left eye was affected and in all of them the eyesight recovered in $1 \frac{1}{2}-3$ months. In the present case both the eyes are affected and recovery has not taken place. 